\title{
Thanatological competence in the psychological education in Russia and abroad
}

\author{
Polina Dmitrieva ${ }^{1, *}$ \\ ${ }^{1}$ Southern Federal University, Rostov-on-Don, Russia
}

\begin{abstract}
The article is devoted to the thanatological formation peculiarities of competence in the education of psychologists in Russia and abroad. The article describes the socio-cultural, professional and individualpsychological relevance of thanatological competence in the implementation of the practical and pedagogical activities of a psychologist. The novelty of the research lies in the substantiation of the need to form thanatological competence as mastering thanatological knowledge for making professional decisions. The article describes the history of the development of "death education" abroad and in Russia, originating in the hospice movement. The article provides a brief description of the didactic and experiential approach to the implementation of programs and courses on the formation of thanatological competence for students in the United States. At the same time, in Russia such courses exist in several higher educational institutions, in others they are presented within the framework of single lectures included in the cycles of basic disciplines. The article contains information about studies demonstrating the need for the formation of thanatological competence in students and the success of the implementation of programs aimed at this abroad. In the conclusion of the article, the prospects for the implementation of programs aimed at the thanatological competence formation for practicing students are described.
\end{abstract}

\section{Introduction}

The relevance of thanatological competence in the context of psychological education, in particular, the education of practicing psychologists and teachers, is due to socio-cultural, professional and individual psychological aspects.

According to many culturologists, sociologists, psychologists, there is a tendency in society to taboo death from the collective consciousness (F. Aries, P.T. Wong, A. Straus, D.M. Rogozin, S.V. Mokhov, A. V. Yurevich and others). Now we can observe the dominance of "personal alienation from death" in society. It manifests itself in the fact that, thanks to the means of mass communication, people are becoming more and more accustomed to seeing the death of strangers, while avoiding thoughts about their own death or the death of their loved ones.

There are two key trends in the problem of death in modern society:

\footnotetext{
*Corresponding author: misspolls@gmail.com
} 
on the one hand, talking about death is mostly avoided, which is confirmed by numerous sociological surveys. Thus, according to a survey conducted in $2013,65 \%$ of respondents prefer not to think about death, $67 \%$ deny the forthcoming death and the necessity to prepare for it. It is prevalent to replace mortal vocabulary with more neutral words such as "passed away", "fell into an eternal sleep", "left the world", and the media actively promotes the image of youth and the fight against aging (Abdulgalimova S.A., Shvareva E.V., Gavrilova T.A.).

on the other hand, death has penetrated popular culture deeply - cinematography, computer games, literature, becoming something ordinary and common, devaluing death as well as life.

Important to note, it is also worth paying attention to the fact that the development of medicine and mass communication has changed the individualization of death. A hundred years ago, the death of loved ones was common due to imperfect medicine, herewith people knew little about the deaths of others. Now, with the development of technology, we can see thousands of deaths: according to the data cited by J. Green (2008), the average American can witness about sixteen thousand deaths in a year through the media and culture (in movies, the Internet, video games) [Mokhov, 2016]. We are surrounded by memorials reminiscent of the war victim, terrorist attacks, and natural disasters. However, this is the death of thousands of people with whom there was no personal acquaintance, and loved ones began to die less often due to the development of medicine, increased life expectancy. Thus, death was distanced from a person, as something that befell "people from television", but not the person himself. Funeral rituals began to lose their relevance and sacred character for people, many of whom do not even know what their meaning is.

V.Yu. Baskakov explains the abundance of mortal themes in cultural works by the need to preserve "the initial balance of the life processes duality", in connection with which the taboo theme of death penetrates into public consciousness in the form of ersatz death [Baskakov, 2005]. Yu.I. Zvonareva connects the penetration of the theme of death into culture with the need for objectification and an attempt to reduce the level of obscurity: "Like anxiety seeking objectification in fear, death, being unknown, is endowed with its own cultural-individual-religious-mystical content and the object of fear." [Zvonareva, 2009, p. 367]

The individual psychological aspect is represented by personal characteristics that are of direct importance in the professional activity of a practicing psychologist: their own position regarding death, the level of anxiety, the level of intensity of psychological defenses associated with the topic of death (especially the mechanisms of denial, displacement, projection). Issues related to death can be encountered in the context of a variety of clients' requests, primarily related to existential crises, issues of grief and loss, neurotic states (such as anxiety-phobic, obsessive-compulsive, provoking panic attacks and manifestate psychosomatic diseases). All of them are widely represented in psychocorrectional and psychotherapeutic practice. Thus, the likelihood of encountering thanatic problems in the professional practice of a psychologist is more than vast. At the same time A.A. Bakanova draws attention to the fact that the professional position of specialists in helping professions, in particular, psychologists, about death is formed in an undirected manner, most often under the influence of personal experience and intuition and is not associated with the deliberate development of relevant knowledge. The overwhelming majority of educational programs for the training of psychologists and teachers do not include the formation of thanatological competence, despite its wide demand in professional activities. In addition, clarifying one's own professional position on death as an existential given is an important prevention of a burnout and secondary trauma.

Thus, the formation of "thanatological competence as mastering thanatological knowledge for making professional decisions" [Trushina, Justus, 2014] is an important aspect 
in the training of education for psychologists and teachers. It is important to pay attention to the fact that, despite its relevance, the term "thanatological competence" in Russia appeared only in the 2000s in the works of T.A. Gavrilova, as well as the works of O. Yu. Trushina and I.V. Justus dedicated to education of medical college graduates.

Based on the aforementioned, the purpose of this article is to review the situation of the thanatological competence formation that exists in Russia and abroad in the framework of the practicing psychologists and teachers' education, and to describe the prospects for implementing programs aimed at its formation.

\section{Methods}

As part of the study, a comparative historical analysis of literary sources devoted to the study of the phenomenon of death as an object of research and pedagogical activity was used in psychological education. In this context, we were interested in studies showing the relevance of thanatological education and the effectiveness of its implementation.

Thanatological research, both theoretical and experimental, has been conducted in Western psychological science since the 70s years of the last century, the beginning of which was the hospice movement (in Russia, the first hospices began to open only in the 90s), which became a response to an impersonal and high-tech way of dealing with death in Western society [Kastenbaum, 1995]. During this time, foreign colleagues had accumulated a large theoretical and empirical material on age, socio-psychological and personal characteristics associated with the specifics of attitudes towards death. The obtained results had wide applied practice and were included in the compulsory training program for specialists in the helping profile - first of all, medical staff, and then psychologists-practitioners, and developed into a whole direction of "death education", combining knowledge of the sciences of the sociohumanitarian cycle about the issues of death, dying, grief and loss. Soon, professional standards and certification programs for specialists in these matters began to develop, the first of which were created through the activities of the Association for Education and Counseling on Matters (established in 1975). Mainly, these programs were developed for medical personnel, but then they entered the professional education of psychologists in the areas of clinical, counseling, children, family and developmental psychology, and began to be included in school curriculum.

At the same time, journals devoted to death and dying began to be published: "Omega: Journal of Death and Dying and Death Studies" (originally called "Death Education", published since 1970), Mortality (published since 1996). In Russia, such journals include "The Archeology of Russian Death" (published since 2015), the materials of which are mainly represented by the results of sociological and anthropological research.

\section{Results}

In the 90s in the US, courses on thanatics also began to be included in the educational programs of secondary schools. Their usefulness had been confirmed by a number of studies that have emphasized the usefulness of open dialogue with children and adolescents about death, forming their adequate view and attitudes about death in the context of media influence. However, such courses were often criticized due to the lack of competent teachers, the lessons with whom not only did not help with understanding issues related to death, but also contributed to an increase in the level of anxiety and traumatization, including suicidal attempts. Research by Mak (2012-2013) also identified insufficient thanatological competence and the need for teachers to develop competence in matters of death, dying and loss. 
Foreign studies (M. Malliarou, P. Sarafis, K. Sotiriadou, 2011; L. Peters, R. Cant, S. Payne, 2013; M. Brady, 2015; P. Thiemann, T. Quince, J. Benson, 2015) show how the psychologist's adequate attitude to death affects the quality of the psychological support provided in crisis situations. A survey conducted by Rosenthal back in the 80 s of the last century showed that $80 \%$ of practicing psychologists note the necessity to study the topic of death as part of their professional training. Based on that data, Worden and Proctor proposed a program to reduce the level of fear of death in future specialists by increasing personal awareness of the issues of dying, death and grief, as well as reducing the level of tension of psychological defenses associated with the least constructive of them: denial, exclusion and projection.

In the USA, thanatological education develops in two aspects: didactic and experiential. The first includes theoretical education in the form of mastering knowledge of death and dying, grief and loss. The experiential aspect is to develop a personal attitude towards these issues through participation in role-plays, training exercises and group discussions. The combination of these two methods in the education of psychologists and teachers contributes, on the one hand, to the formation of thanatological competence, on the other hand, to personal changes that form professionally significant qualities - the ability to talk on topics related to death, a decrease in the tension of psychological defenses, the level of anxiety and fears, increasing the level of awareness and meaningfulness.

T.A. Gavrilova notes that Russian psychology is "the psychology of an immortal personality" [Gavrilova, 2015, p. 1152] due to the ignorance of thanatic issues both in the framework of psychological research and in the context of educational activities in the training of psychologists. So, in the developmental psychology course, thanatic issues are revealed only in the topic of aging and dying, without including the peculiarities of the attitude towards death in other age periods, while death is one of the most important existential categories and is of particularly important in the development of the valuesemantic sphere of the individual, the formation of a meaningful-life strategy and the search for the meaning of life. At the moment, thanatological education in Russia is presented in the framework of the higher education of practicing psychologists, represented by individual lectures within the professional disciplines such as "Clinical psychology", "Developmental psychology". In a number of educational institutions there are disciplines aimed at the formation of thanatological competence: "Thanatology", "Thanatopsychology", "Psychological assistance in crisis situations", "Suicidology", "Psychology of extreme situations", "Psychogerontology and thanatopsychology" (Far Eastern Federal University, Russian State Pedagogical University im. A.I. Herzen, VISU, SU "Dubna", Leningrad State University named after A.S. Pushkin, Nizhny State University named after N.I. Lobachevsky, SFU, SSU, TverSU), however, the vast majority of universities that prepare psychologists follow the first path. Private thanatological courses are an additional source of thanatological competence formation.

The explanation for this situation lies primarily in the insufficient representation of research in national science. In Russia, the sphere of "death studies" is just beginning to develop and is most represented in the cycle of sociology and social anthropology. In psychology, studies of a thanatic sphere are sparse and scattered. According to this T.A. Gavrilova (2015) explains the difficulties of introducing thanatological courses into the educational program for practicing psychologists: in national science, insufficient theoretical and empirical material has been accumulated on the phenomenon of death, and foreign materials cannot be used due to the influence of socio-cultural and religious factors on attitudes towards death, which is emphasized in many foreign and Russian works. Nevertheless, the experience of foreign research is of great importance both for future research and for the formation of thanatological competence today, the need for which is becoming more and more obvious. 
Research conducted by A.A. Bakanova (2014) among Russian psychology students identified the main components included in the development of thanatological competence of future psychologists in accordance with their demand and awareness. Based on the obtained results, the cognitive, activity and value-motivational components were identified. The cognitive component includes theoretical knowledge and views on the issue in the field of humanitarian thanatology and thanatopsychology, the main methodological approaches for understanding the phenomenon of attitudes towards death and providing psychological support in crisis situations. The activity component includes the development of the skills and abilities necessary to help children and adults in crisis and emergency situations related to thanatic issues (existential crisis, non-integrated experience of facing death, experiencing loss and grief, post-suicide, and others). The value-motivational component is associated with the actualization of the psychologist's personal experiences in the process of working with thanatic problems within the framework of professional activity, the development of a personal position on this matter and the integration of one's own experience related to death.

\section{Discussion}

The integration of thanato-thematic courses into educational programs for specialists in helping professions had been repeatedly criticized. Primarily, it is about the specifics of the particular object of discussion - death and attitude to it, which requires the teacher and students to be psychologically ready to discuss this phenomenon, as well as stereotypes associated with it, including gloominess and sadness. On the other hand, due to complexity and specificity of the topic, higher requirements are imposed on the level of training, both professional and personal, since the consequences of a teaching mistake can cost the student not only an insufficient level of formation of thanatological competence, but also mental health. Another aspect of criticism is the risk of increased levels of anxiety about death due to participation in training and discussion of death topics [Hayes, 2010, 2016]. Nevertheless, numerous studies show the importance of introducing thanatological courses into educational programs for training psychologists and psychotherapists. Thus, Wong emphasizes the effectiveness of such programs, which result in a decrease in the level of fear of death and avoidance of topics related to it [Wong, 2017].

Despite the active work on education about death among schoolchildren, students abroad, specialists in helping professions, teachers, there are still many problems. Fonseca \& Testoni (2012) highlight following issues: resistance from teachers or parents; insufficient level of teachers' training; the stereotype that thanatological education and upbringing should take place at home and be implemented by the family, while in most families the issue of dying and death is traditionally ignored and suppressed [Noppe, 2007]. Wass (2004) also notes the problem of education about death among the elderly population, while the issues of death and attitude towards is especially relevant for this age group, and their solution is difficult due to the unspoken rule on ignoring death. Gilbert \& Murray (2007) draw attention to the influence of the media as a way of informal education about death, which, if unreflected, can contribute to an inadequate understanding of death. In view of this, there is an increasing need for the training of qualified teaching personnel who could help students reflect, understand and reconsider the experience of facing death, broadcast by the media.

A.A. Bakanova analyzed the main reasons for refusing to discuss the topic of death. Among them were: the unacceptability of discussing the topic of death ("indecency"), the actualization of negative experiences, the meaninglessness of discussing death due to its inevitability and the impossibility of influencing it, the likelihood of "attracting" death into life [Bakanova, 2014]. The identified reasons can become the key to understanding the specifics of the implementation of educational courses of thanatological content. First of all, the comfortable, trusting atmosphere within the discussion of the topic of death is carried out, 
the need to shift the emphasis from the awareness of one's own mortality to the integration of this knowledge into the experience of living. On the other hand, it is important to show the resourcefulness of the discussion of this problem, which leads to a better understanding of life, its awareness, meaningfulness, and vivid life. The last argument for not discussing the topic of death is an example of magical thinking, that is based on a denial, which helps a person to prevent pain and suffering when faced with death. The danger of this position lies in the probable traumatization when faced with a real death situation (illness or death of friends, loved ones and pets, own illness or threat to life), related to which the task of the teacher is to clarify the expediency and constructiveness of a dialogue on death and dying.

\section{Conclusion}

The analysis allows us to draw the following conclusions, revealing the relevance and prospects for the introduction and further development of thanato-thematic courses in the training of psychologists:

1. The formation of thanatological competence is relevant in the education of practicing psychologists and teachers and should be expressed in the mastery of knowledge and skills in helping people on issues related to death, as well as in developing one's own personal and professional position on this issue. Relevance is due, on the one hand, to the existential nature of the phenomenon of death and the inevitability of a collision with it, which requires understanding; on the other hand, to the broad representation of this phenomenon in the context of the professional activity of a psychologist;

2. At present, the level of knowledge accumulated in national science shows the need for theoretical and empirical research within the framework of thanatics for the introduction of the obtained materials into educational programs, which is especially important in view of the influence of the socio-cultural factor on attitudes towards death;

3. Foreign research and practice can form the basis of educational programs, however, there is a question of translating foreign research, adapting methods for studying the phenomenon of death and the practice of implementing programs for the development of thanatological competence;

4. The implementation of thanato-thematic courses and programs requires special training of pedagogical personnel capable of carrying out educational activities on thanatic issues;

5. The actual direction of research and practice is thanatological education of students and parents, as well as the elderly.

Thus, thanatopsychology is a promising direction of psychological science, practice and education, and the formation of thanatological competence among practicing psychologists and teachers is of paramount importance for the quality of psychological and educational services provided, as well as psychological safety and resilience of the specialists themselves, which is due to changes in social and the cultural sphere of society and the influence of the mass media in particular.

\section{References}

1. A.A. Bakanova, Personality in extreme and crisis situations of life: a collection of scientific articles of the III International scientific-practical conference (Maritime State University, Vladivostok, 2014)

2. A.A. Bakanova, Medical psychology in Russia: electron. scientific Journal 2(37) (2016)

3. T.A. Gavrilova, Economy and society: electronic journal 4(17) (2015)

4. Yu.I. Zvonareva, Psychological Bulletin of the Ural State University 7 (2009) 
5. S.V. Mokhov, The situation with "Death studies" in modern science. NP 4 (2016)

6. E.L. Soldatova, N.Yu. Zhukova, Bulletin of SUSU. Series: Psychology 3 (2018)

7. E.V. Shvareva, Siberian Pedagogical Journal 9 (2010)

8. O.Yu. Trushina, I.V. Justus, Education in the modern world: the role of universities in the socio-economic development of the region: collection of scientific papers of the International Scientific and Methodological Conference (Publishing house "Samara University", Samara, 2014).

9. A.S. Shtanko, D.G. Pirogov, Clinical and medical psychology: research, teaching, practice: electron. scientific journal 1(11) (2016)

10. A.V. Yurevich, Yaroslavl Pedagogical Bulletin 1 (2018)

11. F. Aries, Man in the face of death (Progress Academy, Moscow, 1992)

12. M. Brady, Emergency Nurse 23(4) (2015)

13. J.W. Green, Beyond the Good Death - The Anthropology of Modern Dying (University of Pennsylvania Press, Philadelphia, 2008)

14. L.C. Fonseca, I. Testoni, OMEGA 64(2) (2012) DOI: 10.2190/OM.64.2.d

15. R. Kastenbaum, Psychology of Death («Springer Publishing Company», New York, 2000)

16. J. Hayes, J. Schimel J. Arndt, E.H. Faucher, Psychological bulletin 5(136) (2010)

17. J. Hayes, C.L.P. Ward, I. McGregor, Journal of Personality and Social Psychology 110(1) (2016)

18. M.H.J. Mak, OMEGA-Journal of Death and Dying 2(66) (2012-2013)

19. M. Malliarou, S. Zyga, E. Fradelos, P. Sarafis, American Journal of Nursing Science. Special Issue: Mental Health Care: Aspects, Challenges and Perspectives 4(2-1), 74-77 (2015) doi: 10.11648/j.ajns.s.2015040201.23

20. L. Peters, R. Cant, S. Payne, et al, Open Nurs J. 7, 14-21 (2013)

21. I.C. Noppe, Life span issues and death education. Handbook of thanatology (Routledge, London, 2007)

22. P. Thiemann, T. Quince, J. Benson, D. Wood, S. Barclay, Journal of Pain and Symptom Management 50(3), 335-342 (2015)

23. H.A. Wass, Death Studies 28 (2004)

24. P.T.P. Wong, International Journal of Existential Psychology and Psychotherapy 3(2) (2010)

25. P.T.P. Wong, A. Tomer, Death Studies 35, 2 (2011)

26. P.T.P. Wong, The Humanistic Psychologist 45(3), 207 (2017) 